\title{
Outcomes in Patients With COVID-19 Disease and High Oxygen Requirements
}

\author{
Geurys Rojas-Marte ${ }^{\mathrm{a}, \mathrm{b}}$, Arsalan Talib Hashmi ${ }^{\mathrm{a}}$, Mazin Khalid ${ }^{\mathrm{a}, \mathrm{e}}$, Nnamdi Chukwuka ${ }^{\mathrm{c}}$, Joshua Fogeld, \\ Alejandro Munoz-Martinez ${ }^{c}$, Samantha Ehrlich' ${ }^{\mathrm{c}}$, Maham Akbar Waheed ${ }^{\mathrm{c}}$, Dikshya Sharma ${ }^{\mathrm{c}}$, \\ Shaurya Sharmac ${ }^{c}$, Awais Aslamc, Sabah Siddiquia ${ }^{\mathrm{a}}$, Chirag Agarwala, Yuri Malysheva, \\ Carlos Henriquez-Felipe ${ }^{a}$, Jacob Shani ${ }^{\text {a }}$
}

\begin{abstract}
Background: Approximately $19 \%$ of people infected with the severe acute respiratory syndrome coronavirus 2 (SARS-CoV-2) progress to severe or critical stages of the coronavirus disease 2019 (COVID-19) with a mortality rate exceeding $50 \%$. We aimed to examine the characteristics, mortality rates, intubation rate, and length of stay (LOS) of patients hospitalized with COVID-19 disease with high oxygen requirements (critically ill).
\end{abstract}

Methods: We conducted a retrospective analysis in a single center in Brooklyn, New York. Adult hospitalized patients with confirmed COVID-19 disease and high oxygen requirements were included. We performed multivariate logistic regression analyses for statistically significant variables to reduce any confounding.

Results: A total of 398 patients were identified between March 19th and April 25th, 2020 who met the inclusion criteria, of which 247 $(62.1 \%)$ required intubation. The overall mortality rate in our study was $57.3 \%(\mathrm{n}=228)$. The mean hospital LOS was $19.1 \pm 17.4$ days. Patients who survived to hospital discharge had a longer mean LOS compared to those who died during hospitalization $(25.4 \pm 22.03$ days versus 10.7 \pm 1.74 days). In the multivariate analysis, increased age, intubation and increased lactate dehydrogenase (LDH) were each independently associated with increased odds of mortality. Diarrhea was associated with decreased mortality (OR 0.4 ; CI $0.16,0.99$ ). Obesity and use of vasopressors were each independently associated with increased intubation.

Conclusions: In patients with COVID-19 disease and high oxygen requirements, advanced age, intubation, and higher LDH levels were

Manuscript submitted December 10, 2020, accepted December 21, 2020

Published online January 12, 2021

aDepartment of Cardiology, Maimonides Medical Center, Brooklyn, NY, USA ${ }^{b}$ Donald and Barbara Zucker School of Medicine at Hosftra/Northwell, Staten Island, NY, USA

'Department of Internal Medicine, Maimonides Medical Center, Brooklyn, NY, USA

dDepartment of Business Management, Brooklyn College, Brooklyn, NY, USA ${ }^{e}$ Corresponding Author: Geurys Rojas-Marte and Mazin Khalid, Department of Cardiology, Maimonides Medical Center, 4802 10th Ave, Brooklyn, NY, USA.

Email: gromart005@gmail.com and dr.mazinkhalid@gmail.com

doi: https://doi.org/10.14740/jocmr4405 associated with increased mortality, while diarrhea was associated with decreased mortality. Gender, diabetes, and hypertension did not have any association with mortality or length of hospital stay.

Keywords: COVID-19 disease; SARS-CoV-2; High oxygen requirement; Critically ill

\section{Introduction}

The coronavirus disease 2019 (COVID-19) is the most devastating pandemic of the 21 st century. The first case was detected in Wuhan, China in December 2019 [1]. Since then, the virus has spread globally with an exponential increase in the number of cases. The causative organism, severe acute respiratory syndrome coronavirus 2 (SARS-CoV-2), is a single-stranded ribonucleic acid (RNA) virus that belongs to the Coronaviridae family, and is transmitted mainly by respiratory droplets $[1,2]$. As of December 28th 2020, over 80 million cases have been diagnosed worldwide with more than 13 million cases in the USA [3]. Infection with SARS-CoV-2 ranges from an asymptomatic carrier state to critical illness characterized by acute respiratory distress syndrome (ARDS) with multi-organ failure and death in the most severe cases [4].

Approximately $19 \%$ of people infected with the SARS$\mathrm{CoV}-2$ virus progress to severe or critical COVID-19 disease [4]. Critical illness is characterized by high oxygen requirements that ranges from oxygen supplementation via face mask to intubation and mechanical ventilation. Risk factors associated with development of critical disease include older age, hypertension, diabetes, and obesity [5]. Oxygen levels upon admission and inflammatory markers, including C-reactive protein (CRP) and lactate dehydrogenase (LDH) have been proposed as predictors of poor prognosis in these patients [6].

Mortality in critically ill patients with COVID-19 is extremely high and exceeds $50 \%[4,7]$. Studies from the USA have been limited by the inclusion of a large number of patients who remain hospitalized at the time of the analysis $[5,8]$. We conducted a retrospective analysis to describe the characteristics, mortality, intubation rate, and length of stay (LOS) of patients hospitalized with COVID-19 disease and high oxygen requirement (critically ill) in a single center in Brooklyn, New York. 


\section{Materials and Methods}

\section{Study setting}

We conducted a single center, retrospective, observational analysis at Maimonides Medical Center, a 711-bed tertiary care teaching hospital in Brooklyn, New York. The Maimonides Medical Center Institutional Review Board approved the study as minimal risk research and waived the need for informed consent. This study was conducted in compliance with the ethical standards of the responsible institution on human subjects as well as with the Helsinki Declaration.

We identified patients 18 years of age and older who were admitted between March 19th and April 25th, 2020 with COVID-19 disease and high oxygen requirements. We considered patients to have a high oxygen requirement if they developed acute hypoxemic respiratory failure and required intubation with mechanical ventilation or needed high-level oxygen supplementation (face mask at more than $10 \mathrm{~L}$ per minute, high-flow nasal cannula (HFNC), or non-rebreather (NRB) oxygen face mask) at the time of admission or during hospitalization. The accepted method for diagnosing SARS-CoV-2 infection was real-time reverse transcription-polymerase chain reaction (RT-PCR) of a nasopharyngeal sample. All patients included in this study presented to our emergency department with either symptoms suggestive of COVID-19 infection or had a history of exposure to a person with known COVID-19 infection. For patients who presented with symptoms and had a positive result after being tested as outpatient, they were re-tested in our emergency department to confirm COVID-19 infection. We excluded patients not requiring high concentrations of oxygen, patients who died within 1 day of being admitted, and those who died during their emergency room stay.

\section{Data collection}

Data were manually collected from the hospital's electronic medical record (Sunrise Clinical Manager). The information collected included patients' demographics, presenting symptoms, comorbidities, initial vital signs on admission, pertinent laboratory tests, treatment received for COVID-19 disease, need for vasopressor support, anticoagulation, use of antibiotics for suspected bacterial superinfection, and outcomes, including length of hospital stay, complications, and mortality.

\section{Outcomes}

The primary outcome was in-hospital mortality. Mortality was described as death in hospital following the diagnosis of COVID-19 disease. The secondary outcome was length of hospital stay.

\section{Prediction models}

Based on the clinical demographics, pertinent laboratory re- sults upon admission and the highest values recorded during hospitalization (CRP, ferritin, D-dimer, LDH, troponin, and procalcitonin), and complications including hemodialysis, need for extracorporeal membrane oxygenation (ECMO), bacteremia, and/or fungemia, we developed prediction models for intubation, length of hospital stay, and mortality.

\section{Statistical analysis}

Descriptive statistics of mean and standard deviation were used to express continuous variables. Frequencies and percentages were used to describe categorical variables. Skewed variables were logarithmic-transformed. Troponin had values of 0 , so 0.01 was added to all values and then the values underwent logarithmic transformation. For intubation, analysis of variance was used to compare the continuous variables and the Pearson's Chisquare test compared the categorical variables except for when expected cell size was $<5$ in which case Fisher's exact test was performed. Any variable that was statistically significant in the univariate analysis was included in the multivariate logistic regression analysis. Univariate logistic regression was conducted for the outcome variable of mortality. Any variable statistically significant in the univariate analysis for mortality was included in the multivariate analysis. Univariate linear regression was conducted for the outcome variable of LOS. Any variable statistically significant in the univariate analysis for LOS was included in the multivariate analysis. All P values were two tailed. Alpha level for significance was at $\mathrm{P}<0.05$. IBM SPSS Statistics Version 26 was used for all analyses (IBM, 2019).

\section{Results}

\section{Baseline characteristics}

A total of 398 critically ill patients with COVID-19 disease were included in this study. Two-hundred forty-seven (62.1\%) patients required intubation, and 151 (37.9\%) needed oxygen supplementation via HFNC or NRB oxygen mask. The mean age was 65.8 years \pm 16.26 , and $52.8 \%$ of patients were 65 years or older. Two thirds of the patients $(66.6 \%)$ were males, while $19.1 \%$ Hispanic. Hypertension was the most common comorbidity, $(n=237 ; 59.5 \%)$ followed by obesity $(n=167$; $42.0 \%)$, and diabetes mellitus $(\mathrm{n}=141 ; 35.4 \%)$. Other comorbidities included coronary artery disease (CAD) at $14.1 \%$, heart failure at $11.3 \%$, atrial fibrillation at $9.8 \%$, and chronic obstructive pulmonary disease (COPD) at $6.8 \%$. The most common presenting symptoms included shortness of breath $(83.2 \%)$, fever $(73.4 \%)$, and cough $(70.9 \%)$. Patients' demographics, comorbidities, presenting symptoms, and laboratory values are given in Table 1. Additional baseline characteristics are shown here (Supplementary Material 1, www.jocmr.org).

\section{Management}

Most patients received hydroxychloroquine (93.7\%) and 

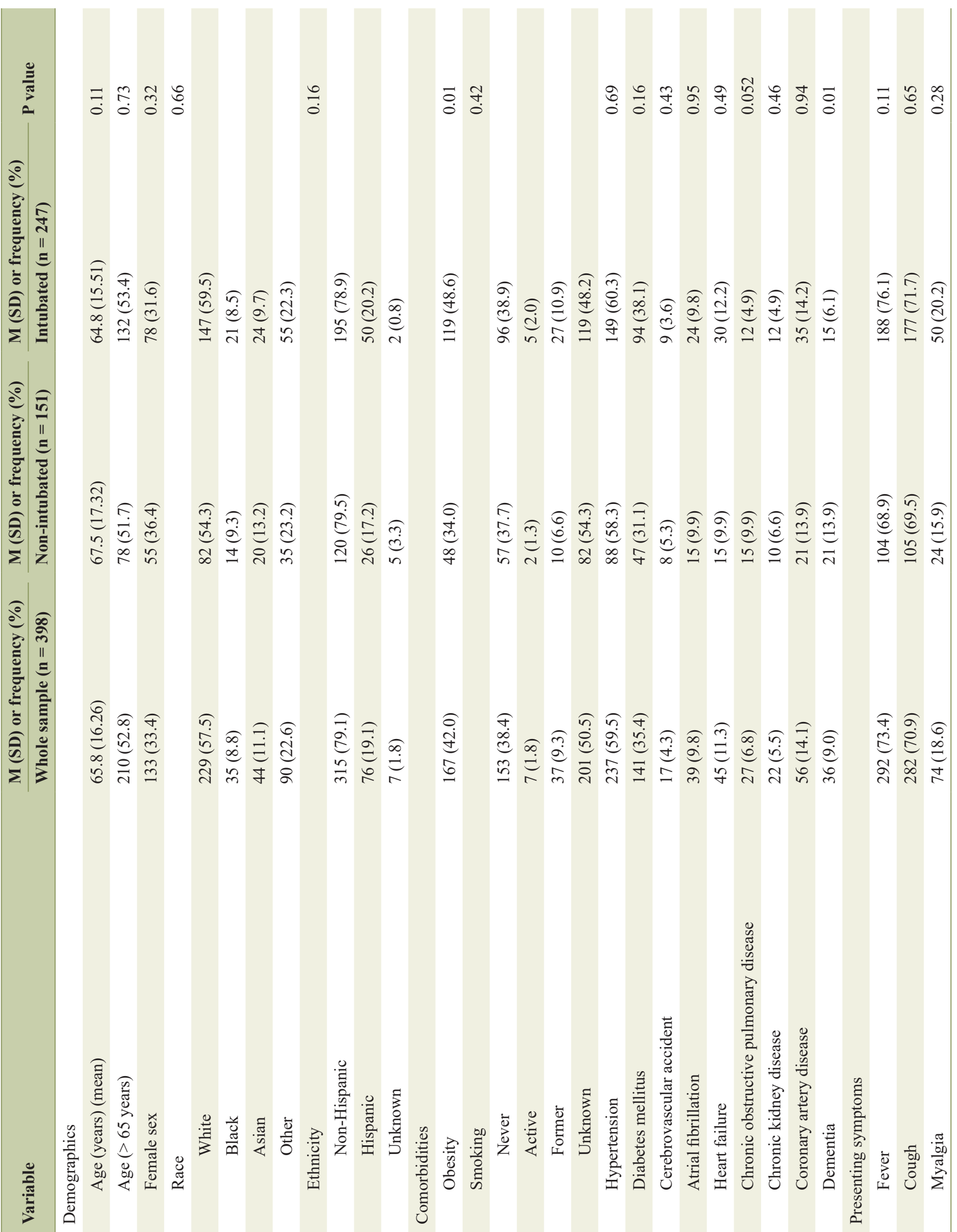


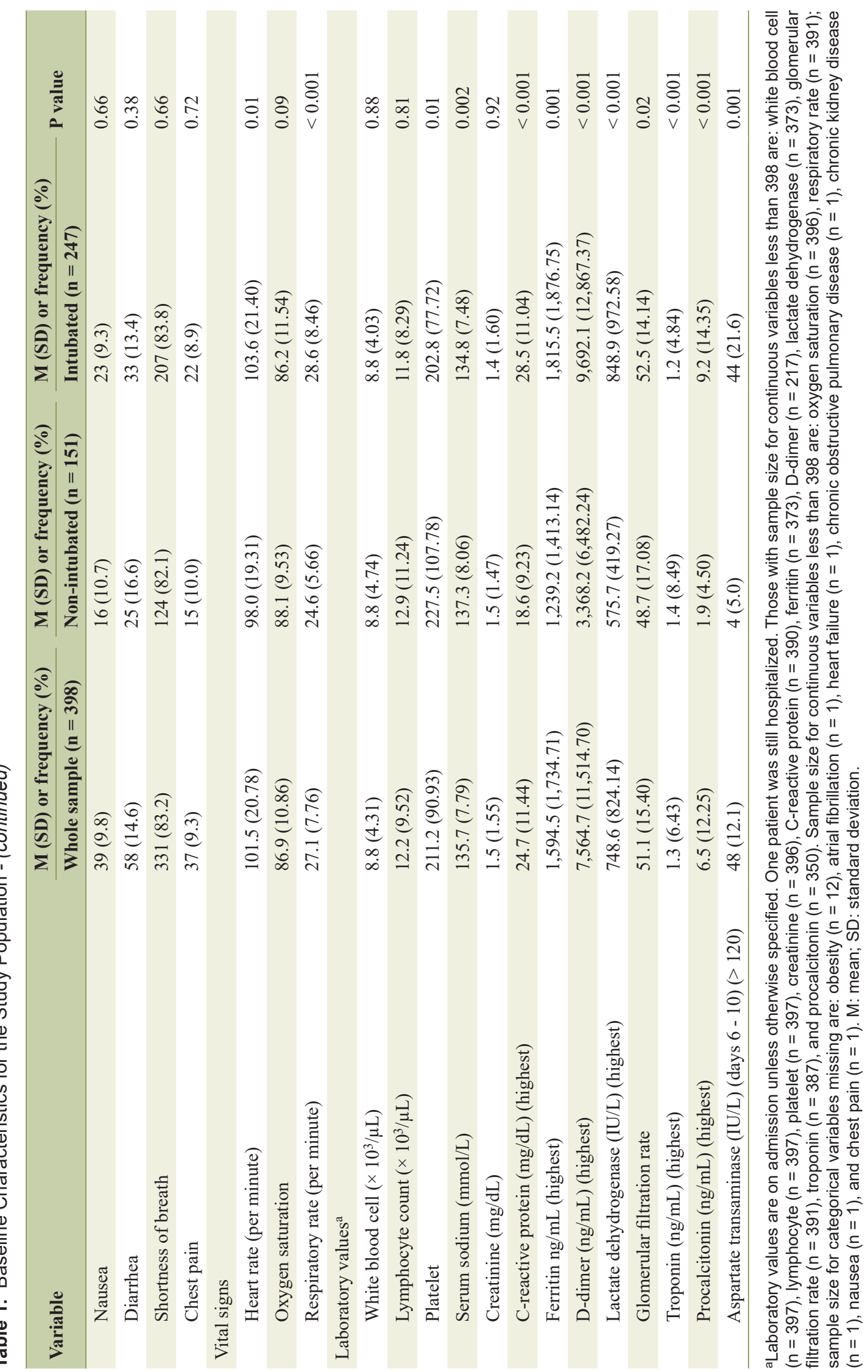


azithromycin (93.2\%). Tocilizumab was given to $29.6 \%$ of the patients while $9.8 \%$ received remdesivir. Convalescent plasma was given to $7.5 \%$ of patients, while $39.2 \%$ received full dose anticoagulation. Table 2 summarizes the treatments received during admission.

\section{Complications}

More than half of the patients $(57.8 \%)$ needed vasopressor support. Hemodialysis was used in one-fifth of patients while blood transfusion was administered to $20.9 \%$. Bacteremia and/ or fungemia were documented in one-fifth of the cases. The majority of patients $(91.5 \%)$ received antibiotics (Table 2). Figure 1 shows the percentage of some of the complications observed in the study population.

\section{Predictors of intubation}

Tables 1 and 2 show univariate comparisons for those patients who were either intubated or nonintubated. In the multivariate analysis, only obesity and use of vasopressor were each independently associated with increased odds for intubation (Table 3).

\section{Predictors of mortality}

Overall mortality in our study was $57.3 \%(n=228)$. In an analysis comparing intubation and mortality, the mortality rate was significantly higher in intubated patients $(78.1 \%)$ as compared to those not intubated $(23.2 \%)$. Table 4 shows logistic regression analyses for mortality. In the multivariate analysis, increased age, intubation, and increased LDH were each independently associated with increased odds of mortality. Diarrhea was independently associated with decreased mortality. None of the comorbidities (including diabetes and hypertension), vital signs, treatment management, or complications was significantly associated with mortality.

\section{Predictors of LOS}

The mean hospital LOS for the entire cohort was $19.1 \pm 17.4$ days. In the subset of patients who were discharged alive, mean LOS was $25.4 \pm 22.03$ days. Patients who died during hospitalization had a mean LOS of $10.7 \pm 1.74$ days. In an analysis comparing intubation and LOS, intubated patients had significantly greater mean LOS $(21.7 \pm 19.08$ days $)$ as compared to those not intubated (14.8 \pm 12.67 days) as shown in Table 2 .

Table 5 shows the linear regression analyses for LOS. In the multivariate analysis, Asian race, hemodialysis, ECMO support, blood transfusion, treatment with steroids, remdesivir, or vitamin $\mathrm{C}$, and diagnosis of bacteremia and/or fungemia were each independently associated with increased LOS. CAD was significantly associated with decreased LOS. In a multivariate analysis in the subset of patients who survived to dis- charge for variables significant in the whole sample multivariate analysis, hemodialysis, blood transfusion, use of vitamin C, and diagnosis of bacteremia/fungemia were each significantly associated with increased LOS. In the subset of patients with mortality for those variables significant in the whole sample multivariate analysis, blood transfusion, treatment with steroids, remdesivir, or vitamin $\mathrm{C}$ were each significantly associated with increased LOS. CAD was significantly associated with decreased LOS.

\section{Discussion}

Our study represents one of the largest analyses of patients with COVID-19 disease with high oxygen requirements and complete outcomes. We included 398 critically ill patients, of which 247 (62.1\%) were intubated. The reported percentage of intubation in COVID-19 patients with critical disease ranges from $57 \%$ to $88 \%$ [7, 9-12]. We found obesity and use of vasopressors were each independently associated with higher odds of intubation. These findings are consistent with a study that reported higher rates of intubation in obese COVID-19 patients [10].

The mean LOS in our study population was 19.1 days. Other studies on critically patients with COVID-19 have reported a $\operatorname{LOS}$ of 15 - 22 days $[5,10]$. We found that patients who died had a 2.5-time shorter LOS as compared to those who lived. This finding is consistent with other studies that have reported LOS for patients who died between 4 and 21 days and 4 to 53 days for those who were discharged alive [13].

The overall mortality in our cohort was $57.3 \%$. This finding was mainly driven by a significantly higher mortality in intubated patients than non-intubated patients $(78.1 \%$ versus $23.2 \%$ ). In a previous study addressing patients with COVID-19, we reported a mortality of $50 \%$; however, that study included patients with less severe disease [14]. Our current findings are consistent with reports from China concerning critically ill patients with COVID-19 disease. A study that examined 239 critically ill patients, of which $69 \%$ were intubated, showed a $61.5 \%$ mortality [7]. Similarly, another group reported a mortality rate of $56 \%$ in which more than half of the studied patients were intubated [9]. In a study from Italy that included 1,300 patients with COVID-19 treated in the intensive care unit (ICU), mortality was $26 \%$ despite $88 \%$ of the patients being intubated. However, more than $50 \%$ of patients remained hospitalized at the time of the analysis [15].

Studies from the New York City area have included cohorts of similar illness severity as our sample. The mortality rate reported in these studies ranged from $39 \%$ to $68 \%$. However, they included a high percentage of patients (ranging from $23 \%$ to $37 \%$ ) who remained hospitalized at the time of the analysis, which makes their results on mortality and LOS inconclusive $[8,11,16]$. In our study, only one patient remained hospitalized under hospice care.

Various authors examined predictors of mortality and many reported older age to be independently associated with increased mortality $[7,11]$. Other factors associated with higher mortality in critically ill patients with COVID-19 include chronic cardiac 


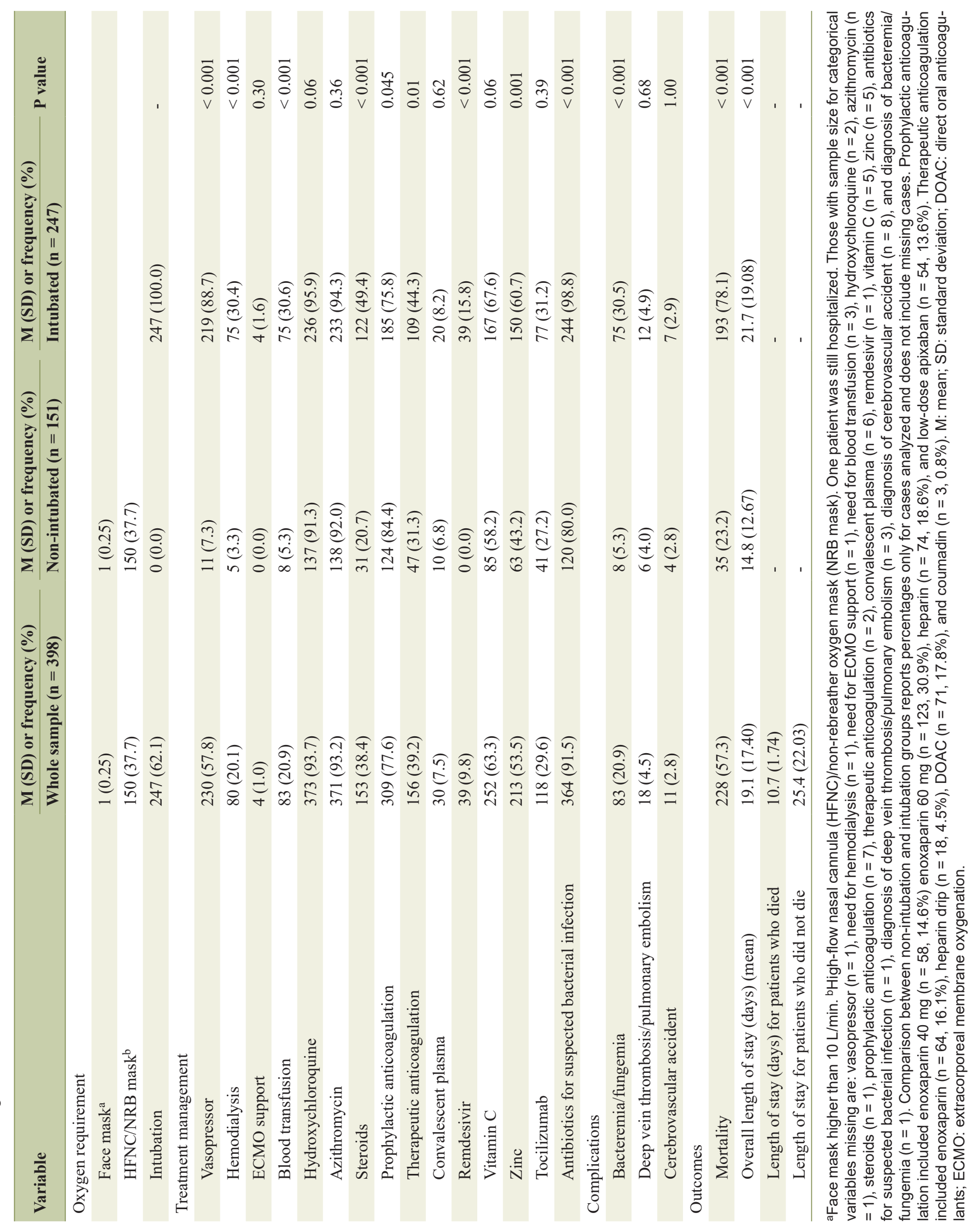


Table 3. Logistic Regression for Intubation

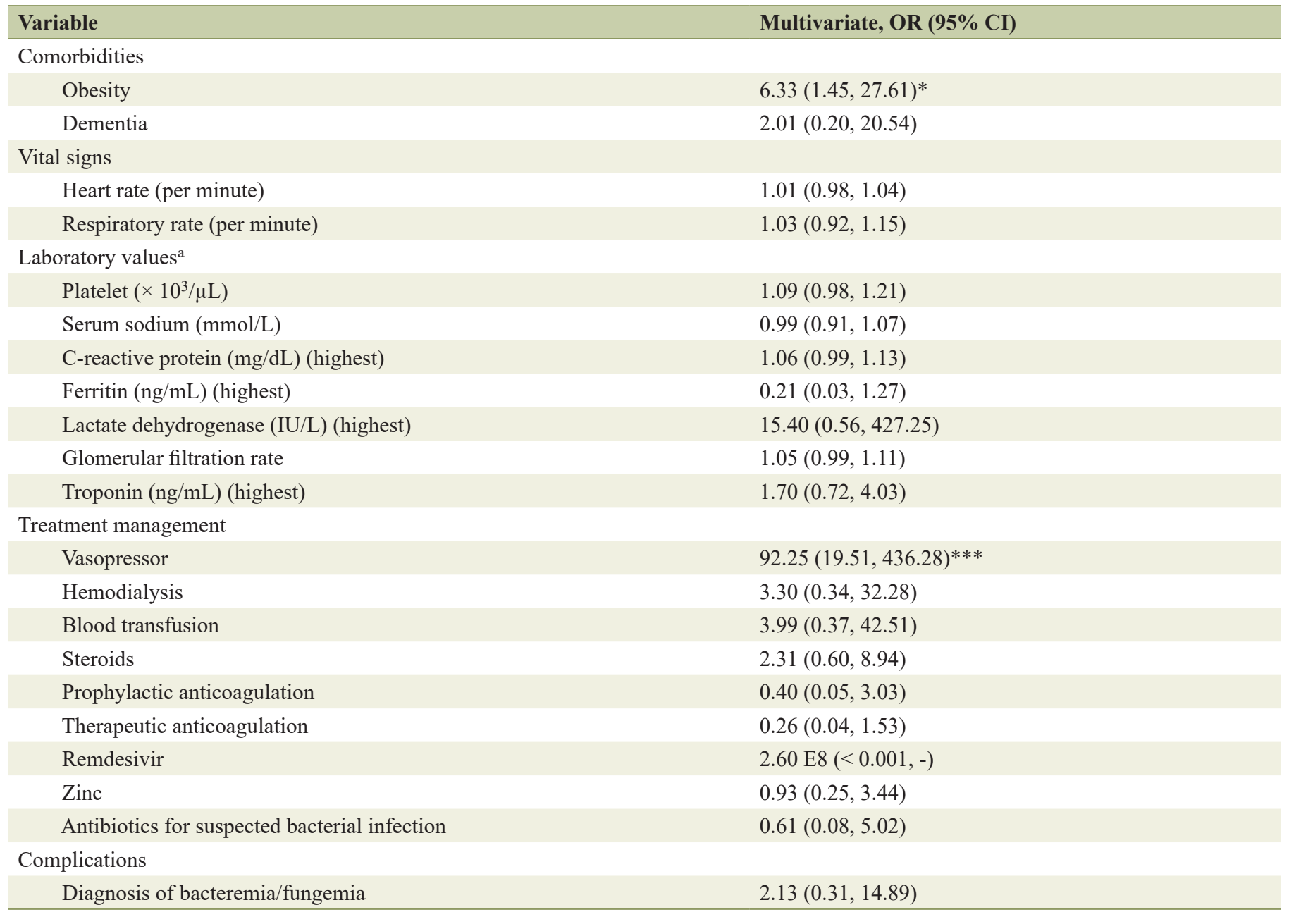

${ }^{*} \mathrm{P}<0.05,{ }^{* * *} \mathrm{P}<0.001$. Analysis included one patient still hospitalized. Analysis includes 238 patients due to missing data. D-dimer, procalcitonin, and aspartate transaminase were not included in the multivariate analysis due to a lot of missing data. Nagelkerke R Square $=0.82$. ${ }^{2}$ aboratory values are on admission unless otherwise indicated. OR: odds ratio; $\mathrm{Cl}$ : confidence interval.

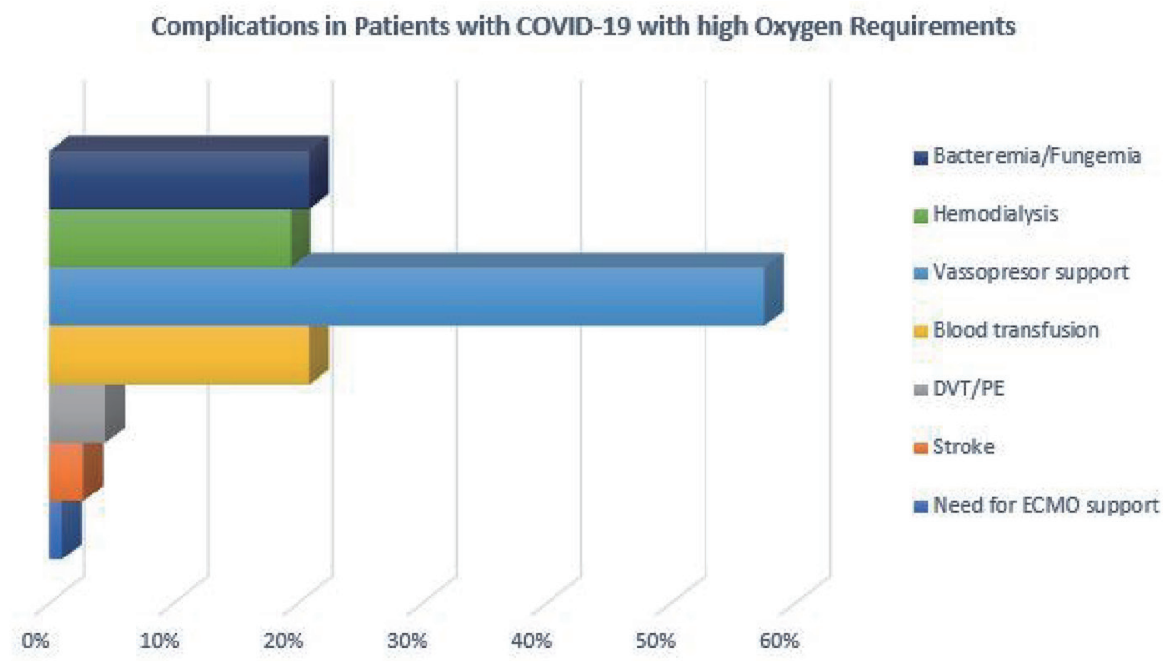

Figure 1. Complications in patients with COVID-19 disease and high oxygen requirements. 
Table 4. Mortality Analysis

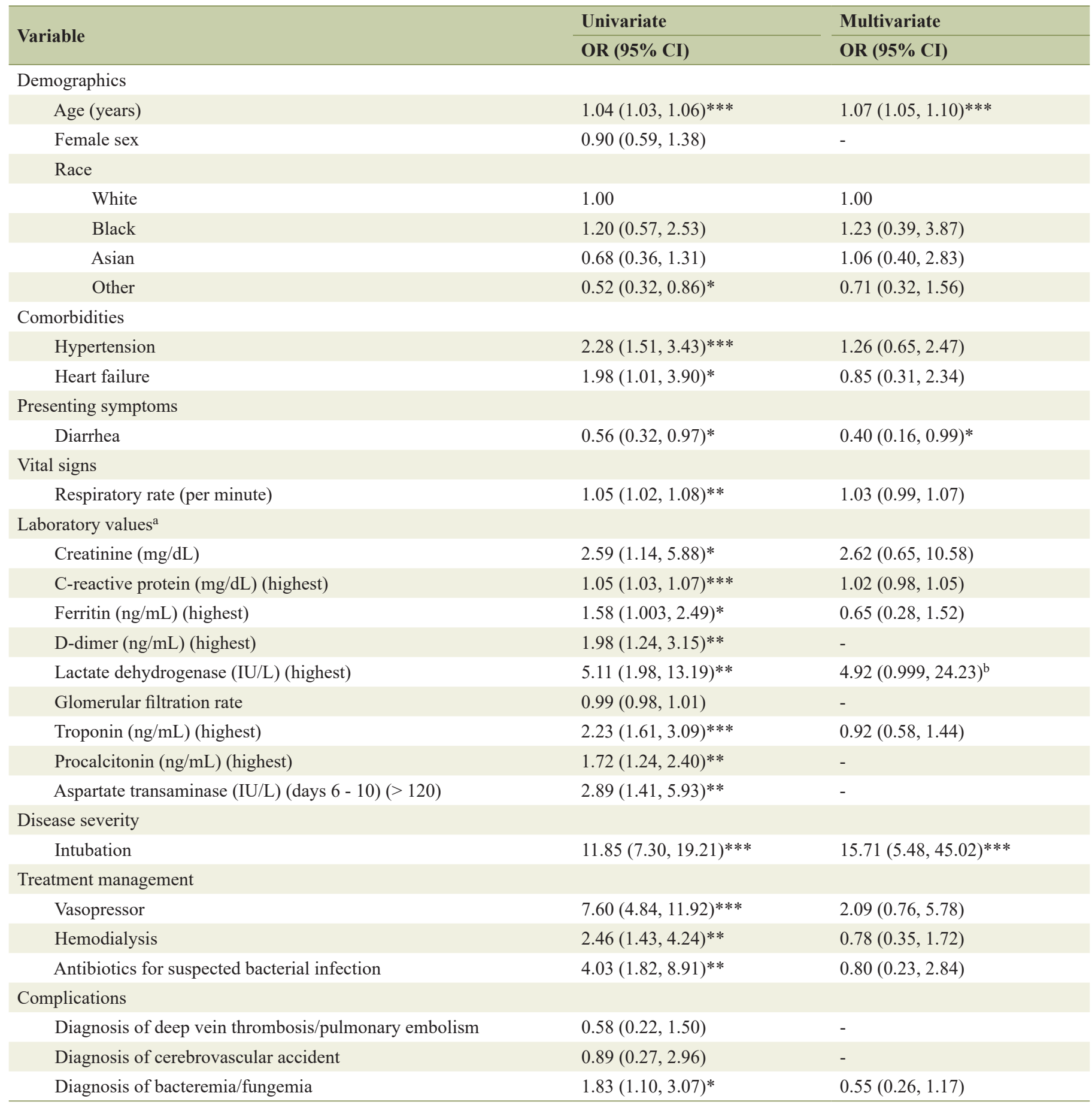

aLaboratory values are on admission unless otherwise indicated. ${ }^{b} \mathrm{P}=0.05,{ }^{*} \mathrm{P}<0.05,{ }^{* *} \mathrm{P}<0.01,{ }^{* * *} \mathrm{P}<0.001$. Analysis included one patient still hospitalized. Multivariate analysis includes 333 patients due to missing data. D-dimer, procalcitonin, and aspartate transaminase were not included in the multivariate analysis due to missing data. Nagelkerke R Square $=0.54$. OR: odds ratio; Cl: confidence interval. For brevity purposes, many variables not statistically significant in the univariate analyses are not shown in the table.

disease (CAD and heart failure combined), high concentration of interleukin-6, elevated D-dimer, thrombocytopenia, acute kidney injury, and ARDS [7, 11, 17]. We found increased age, LDH levels and intubation were each independently associated with increased mortality. In a pooled analysis including 1,532 patients, LDH levels were found to be associated with a 16-fold increase in mortality [18]. That analysis was based on the LDH value upon admission, whereas in our study we used the highest 

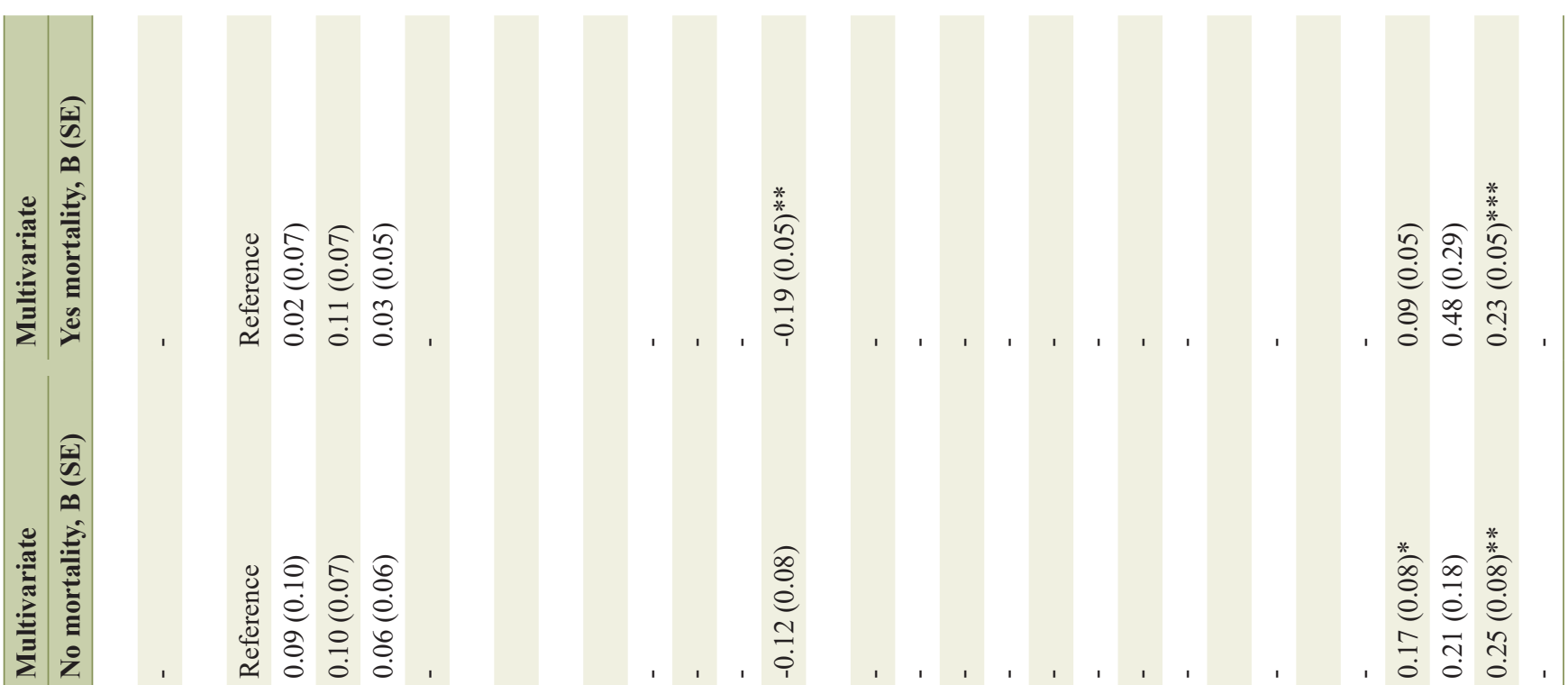

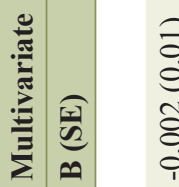
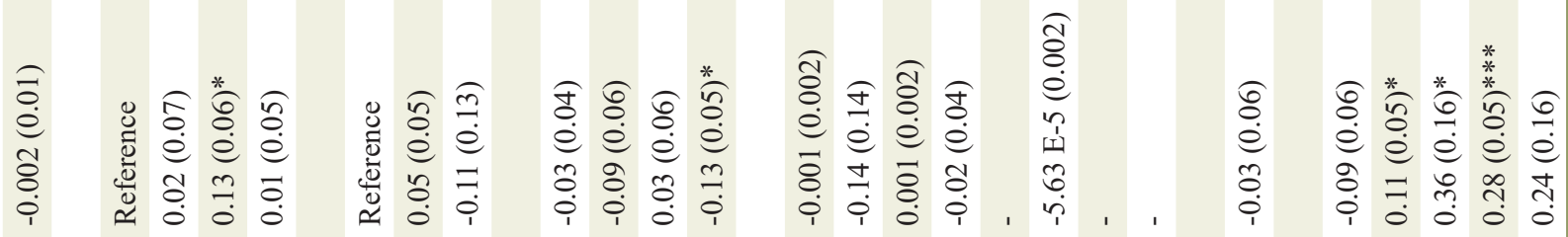

竎
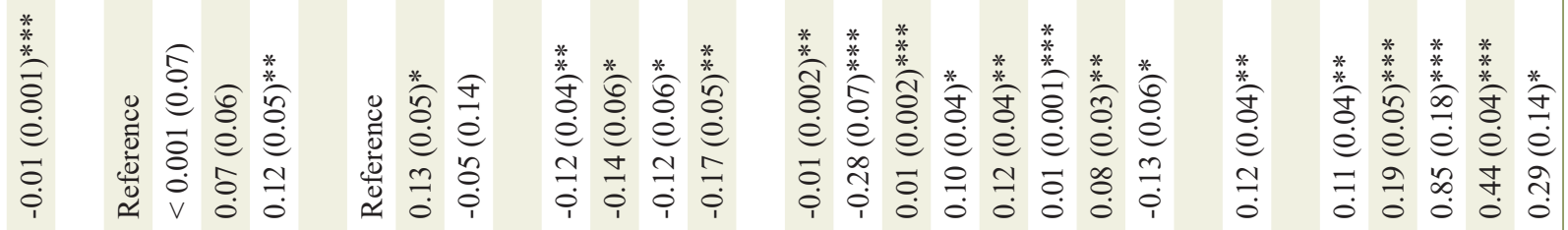

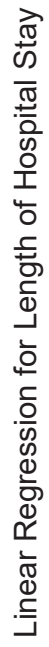

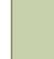




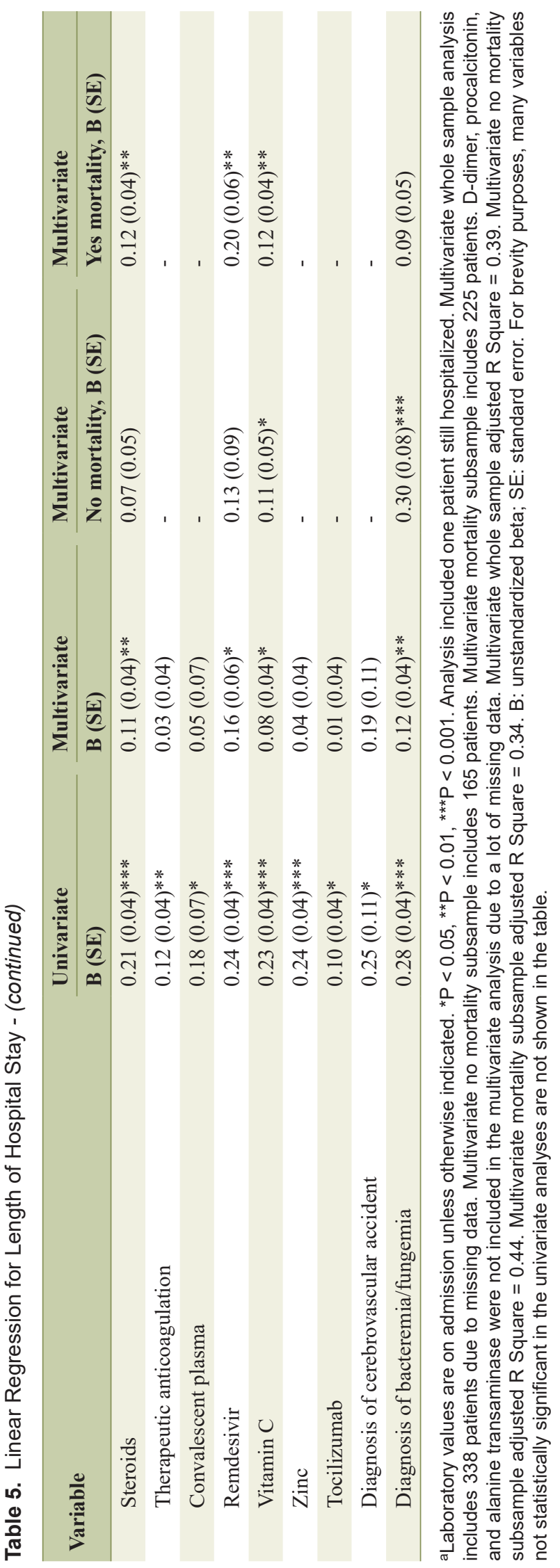

LDH value during hospitalization.

Even though diabetes mellitus and hypertension are recognized risk factors for developing severe COVID-19 disease, they have failed to consistently show any association with higher mortality in critically ill patients $[17,19,20]$. One study found no difference in the rate of hypertension or diabetes between survivors and non-survivors of critical COVID-19 disease [7]. In contrast, another study reported a significant difference in mortality in patients with severe COVID-19 disease with diabetes versus non-diabetic patients ( $81 \%$ versus $47 \%)$. However, this difference in outcome could also be explained by the 10-year difference in age between the groups in that study [9]. Nonetheless, despite having a lower rate of hypertension and diabetes as compared to our cohort, both studies reported similar number of intubated patients and mortality rate similar to our findings $[7,9]$.

A study from Detroit, Michigan in the USA reported a mortality rate of $39 \%$ in 141 patients with COVID-19 who were treated in the ICU, including $114(80 \%)$ intubated patients. Their cohort included $51.8 \%$ with diabetes and $78.7 \%$ with hypertension and yet the reported mortality was significantly lower than other reports with COVID-19 patients of similar severity [10]. These studies, including ours, suggest that diabetes and hypertension might not affect mortality in patients with COVID-19 disease once they progress to critical illness. Similarly, we did not find any association between gender and mortality. This finding is consistent with previously published data [7].

Previous studies examined gastrointestinal involvement in COVID-19 and reported diarrhea to be associated with prolonged symptoms, viral carriage, development of cytokine storm, and multi-organ damage [21, 22]. Other researchers have failed to establish an association between gastrointestinal symptoms, including diarrhea, with increased mortality, LOS, or mechanical ventilation [23]. Our analysis showed diarrhea to be independently associated with decreased mortality.

\section{Limitations}

Our study has some limitations. First, our analysis is subject to data entry errors given that the information was manually collected. To minimize this, we performed multiple checks during the process of data collection. Prior to data analysis, we double checked very abnormal laboratory values. Second, we did not include mechanical ventilation settings, which could be important information for management of these patients. However, our objective was to focus on mortality and LOS outcome. Third, smoking information was self-reported and was not provided in over $50 \%$ of patients. This information could be an important risk factor in patients with COVID-19 disease.

\section{Conclusions}

Our study shows a high mortality rate in COVID-19 patients with high oxygen requirements. This finding is driven mainly 
by higher mortality in intubated patients. We found a significant difference in LOS between patients who died during hospitalization as compared to those who survived to discharge. Older age, intubation, and higher LDH levels were associated with increased mortality, while diarrhea was associated with decreased mortality. Gender, diabetes, and hypertension did not have any association with mortality or length of hospital stay.

\section{Supplementary Material}

Suppl 1. Complete list of baseline characteristics.

\section{Acknowledgments}

None to declare.

\section{Financial Disclosure}

This study did not receive any grant from any funding agency in the public, commercial, or not-for-profit sectors.

\section{Conflict of Interest}

None to declare.

\section{Informed Consent}

Informed consent was not needed due to the retrospective nature of the study and data anonymization.

\section{Author Contributions}

All the authors reviewed the manuscript and agreed with the findings and interpretation. Geurys Rojas-Marte: conception and design, supervision, drafting of the manuscript, critical review, review of data integrity and final approval. Arsalan Talib Hashmi: supervision, review of data integrity, critical review. Mazin Khalid, Nnamdi Chukwuka: drafting the manuscript, scientific writing, critical review, and content approval. Joshua Fogel: review of data and statistical analysis. Alejandro MunozMartinez, Samantha Ehrlich, Maham Akbar Waheed, Dikshya Sharma, Shaurya Sharma, Awais Aslam, Sabah Siddiqui, Chirag Agarwal, Yuri Malyshev, Carlos Henriquez-Felipe: data acquisition. Jacob Shani: final approval of publication, critical review.

\section{Data Availability}

The authors declare that data supporting the findings of this study are available within the article.

\section{References}

1. Epidemiology Working Group for Ncip Epidemic Response, Chinese Center for Disease Control Prevention. [The epidemiological characteristics of an outbreak of 2019 novel coronavirus diseases (COVID-19) in China]. Zhonghua Liu Xing Bing Xue Za Zhi. 2020;41(2):145-151.

2. Chan JF, To KK, Tse H, Jin DY, Yuen KY. Interspecies transmission and emergence of novel viruses: lessons from bats and birds. Trends Microbiol. 2013;21(10):544555.

3. Center JHCR. Johns Hopkins Coronavirus Resource Center.

4. $\mathrm{Wu} \mathrm{Z}, \mathrm{McGoogan} \mathrm{JM}$. Characteristics of and Important Lessons From the Coronavirus Disease 2019 (COVID-19) Outbreak in China: Summary of a Report of 72314 Cases From the Chinese Center for Disease Control and Prevention. JAMA. 2020;323(13):1239-1242.

5. Argenziano MG, Bruce SL, Slater CL, Tiao JR, Baldwin MR, Barr RG, Chang BP, et al. Characterization and clinical course of 1000 patients with coronavirus disease 2019 in New York: retrospective case series. BMJ. 2020;369:m1996.

6. Pan F, Yang L, Li Y, Liang B, Li L, Ye T, Li L, et al. Factors associated with death outcome in patients with severe coronavirus disease-19 (COVID-19): a case-control study. Int J Med Sci. 2020;17(9):1281-1292.

7. Xu J, Yang X, Yang L, Zou X, Wang Y, Wu Y, Zhou T, et al. Clinical course and predictors of 60-day mortality in 239 critically ill patients with COVID-19: a multicenter retrospective study from Wuhan, China. Crit Care. 2020;24(1):394.

8. Petrilli CM, Jones SA, Yang J, Rajagopalan H, O'Donnell L, Chernyak Y, Tobin KA, et al. Factors associated with hospital admission and critical illness among 5279 people with coronavirus disease 2019 in New York City: prospective cohort study. BMJ. 2020;369:m1966.

9. Yan Y, Yang Y, Wang F, Ren H, Zhang S, Shi X, Yu X, et al. Clinical characteristics and outcomes of patients with severe covid-19 with diabetes. BMJ Open Diabetes Res Care. 2020;8(1):e001343.

10. Suleyman G, Fadel RA, Malette KM, Hammond C, Abdulla H, Entz A, Demertzis Z, et al. Clinical characteristics and morbidity associated with coronavirus disease 2019 in a series of patients in metropolitan detroit. JAMA Netw Open. 2020;3(6):e2012270.

11. Cummings MJ, Baldwin MR, Abrams D, Jacobson SD, Meyer BJ, Balough EM, Aaron JG, et al. Epidemiology, clinical course, and outcomes of critically ill adults with COVID-19 in New York City: a prospective cohort study. Lancet. 2020;395(10239):1763-1770.

12. Yang X, Yu Y, Xu J, Shu H, Xia J, Liu H, Wu Y, et al. Clinical course and outcomes of critically ill patients with SARS-CoV-2 pneumonia in Wuhan, China: a single-centered, retrospective, observational study. Lancet Respir Med. 2020;8(5):475-481.

13. Rees EM, Nightingale ES, Jafari Y, Waterlow NR, Clifford S, CA BP, Group CW, et al. COVID-19 length of 
hospital stay: a systematic review and data synthesis. BMC Med. 2020;18(1):270.

14. Rojas-Marte G, Khalid M, Mukhtar O, Hashmi AT, Waheed MA, Ehrlich S, Aslam A, et al. Outcomes in patients with severe COVID-19 disease treated with tocilizumab: a case-controlled study. QJM. 2020;113(8):546-550.

15. Grasselli G, Zangrillo A, Zanella A, Antonelli M, Cabrini L, Castelli A, Cereda D, et al. Baseline characteristics and outcomes of 1591 patients infected with SARS-CoV-2 admitted to ICUs of the Lombardy Region, Italy. JAMA. 2020;323(16):1574-1581.

16. Richardson S, Hirsch JS, Narasimhan M, Crawford JM, McGinn T, Davidson KW, the Northwell C-RC, et al. Presenting characteristics, comorbidities, and outcomes among 5700 patients hospitalized with COVID-19 in the New York City Area. JAMA. 2020;323(20):2052-2059.

17. Al-Salameh A, Lanoix JP, Bennis Y, Andrejak C, Brochot E, Deschasse G, Dupont H, et al. Characteristics and outcomes of COVID-19 in hospitalized patients with and without diabetes. Diabetes Metab Res Rev. 2020:e3388.

18. Henry BM, Aggarwal G, Wong J, Benoit S, Vikse J, Plebani M, Lippi G. Lactate dehydrogenase levels predict coronavirus disease 2019 (COVID-19) severity and mortality:
A pooled analysis. Am J Emerg Med. 2020;38(9):17221726.

19. Guan WJ, Liang WH, Zhao Y, Liang HR, Chen ZS, Li YM, Liu XQ, et al. Comorbidity and its impact on 1590 patients with COVID-19 in China: a nationwide analysis. Eur Respir J. 2020;55(5):2001227.

20. Yang J, Zheng Y, Gou X, Pu K, Chen Z, Guo Q, Ji R, et al. Prevalence of comorbidities and its effects in patients infected with SARS-CoV-2: a systematic review and metaanalysis. Int J Infect Dis. 2020;94:91-95.

21. Wei XS, Wang X, Niu YR, Ye LL, Peng WB, Wang ZH, Yang WB, et al. Diarrhea is associated with prolonged symptoms and viral carriage in corona virus disease 2019. Clin Gastroenterol Hepatol. 2020;18(8):1753-1759 e1752.

22. Zhang L, Han C, Zhang S, Duan C, Shang H, Bai T, Hou $\mathrm{X}$. Diarrhea and altered inflammatory cytokine pattern in severe coronavirus disease 2019: Impact on disease course and in-hospital mortality. J Gastroenterol Hepatol. 2020.

23. Ramachandran P, Onukogu I, Ghanta S, Gajendran M, Perisetti A, Goyal H, Aggarwal A. Gastrointestinal symptoms and outcomes in hospitalized coronavirus disease 2019 patients. Dig Dis. 2020;38(5):373-379. 